\title{
g-Steiner, co-Steiner and Normal Points of Bounded Euclidean Submanifolds
}

\author{
Bang-Yen Chen \\ (Communicated by Cihan Özgür)
}

\begin{abstract}
The original "Steiner point" introduced in $\mathbf{1 8 3 8}$ by the Swiss mathematician Jakob Steiner (17961863), also known as the "Steiner curvature centroid", is the geometric centroid of the system obtained by placing a mass equal to the magnitude of the exterior angle at each vertex of a triangle. Steiner points have been studied and applied to networks, combinatorics, computational geometry and even in game theory.

In this article, we extend the notion of Steiner points to the notion of g-Steiner points for bounded Euclidean submanifolds with arbitrary codimension. In this article, we also introduce the notions of co-Steiner and normal points for bounded Euclidean submanifolds. We prove several fundamental properties for such points. Furthermore, we establish some links between g-Steiner, co-Steiner and normal points.
\end{abstract}

\section{Introduction}

The name of "Steiner point" was named after Swiss mathematician Jakob Steiner (1796-1863). The Steiner point, also known as the Steiner curvature centroid, is originally defined to be the geometric centroid of the system obtained by placing a mass equal to the magnitude of the exterior angle at each vertex of a triangle (cf. $[19,20])$. Since then Steiner points have been studied and applied to networks, combinatorics, computational geometry and game theory (cf. e.g., [12, 13, 15, 16, 17]).

Throughout this article, by a bounded manifold we mean a compact manifold with or without smooth boundary. By a closed manifold we mean a bounded manifold without boundary.

For an even-dimensional convex closed hypersurface $M^{n}$ in a Euclidean $(n+1)$-space $\mathbb{E}^{n+1}$, H. Flanders proved in [11] that the Steiner point of $M^{n}$ can be defined as

$$
s\left(M^{n}\right)=\frac{1}{c_{n}} \int_{p \in M^{n}} \mathbf{x} K(p) d v,
$$

where x denotes the position vector field of $M^{n}$ in $\mathbb{E}^{n+1}, d v$ is the volume element of $M^{n}$, and $K(p)$ denotes the Gauss-Kronecker curvature of $M^{n}$ at a point $p \in M^{n}$.

It is known that the Steiner point defined by (1.1) satisfies the following properties (cf. [17, 18]):

- $s\left(a M^{n}\right)=a s\left(M^{n}\right)$ for any similar transformation $a$;

- $s\left(M^{n}+c\right)=s\left(M^{n}\right)+c$ for a constant vector $c \in \mathbb{E}^{n+1}$;

- $s\left(M^{n}\right)$ is a continuous function of $M^{n}$;

- If $\operatorname{dim} M^{n}$ is positive, then $s\left(M^{n}\right)$ is a relative interior point of $M^{n}$.

In this article, we extend the notion of Steiner points to the notion of g-Steiner points for bounded Euclidean submanifolds with arbitrary codimension. We also introduce the notions of co-Steiner and normal points for 
bounded Euclidean submanifolds via the notion of $G$-total curvatures introduced in [4, 7]. In this article, we also prove several fundamental properties for such points. Furthermore, we establish some links between g-Steiner, co-Steiner and normal points.

\section{Preliminaries}

In this article, we follow the notations from $[7,8,9,10]$.

Let $M^{n}$ be a bounded manifold of dimension $n$ and let $\varphi: M^{n} \rightarrow \mathbb{E}^{m}$ be an immersion of $M^{n}$ into an oriented Euclidean space $\mathbb{E}^{m}$ of dimension $m$. By a frame $\left\{p, e_{1}, \ldots, e_{m}\right\}$ in the space $\mathbb{E}^{m}$, we mean a point $p \in \mathbb{E}^{m}$ together with an ordered set of mutually perpendicular unit vectors $e_{1}, \ldots, e_{m}$ whose orientation is coherent with that of the Euclidean space $\mathbb{E}^{m}$. In the following, we shall identify $e_{i}$ with its image $\varphi_{*}\left(e_{i}\right)$ under the differential map $\varphi_{*}$ of the immersion $\varphi$.

Let $\mathcal{F}\left(\mathbb{E}^{m}\right)$ be the set of all frames on the Euclidean $m$-space space $\mathbb{E}^{m}$. We let $\mathcal{F}\left(M^{n}\right)$ denote the set of all orthonormal frames in $M^{n}$ (with respect to the induced metric on $M^{n}$ ) such that $e_{1}, \ldots, e_{n}$ are tangent to $M^{n}$ and hence $e_{n+1}, \ldots, e_{m}$ are normal to $M^{n}$.

Let us denote by $B_{1}(\varphi)$ the bundle space of unit normal vectors of $\varphi\left(M^{n}\right)$ so that a point in $B_{1}(\varphi)$ is a pair $(p, e)$, where $e$ is a unit normal vector of $M^{n}$ at $\varphi(p)$. Then $B_{1}(\varphi)$ forms a principal bundle of $(m-n-1)$ dimensional unit-spheres $S_{p}^{m-n-1}$ at $p \in M^{n}$. Clearly, $B_{1}(\varphi)$ is a manifold of dimension $m-1$. Let $B(\varphi)$ denote the set consists of all $b=\left(p, e_{1}, \ldots, e_{m}\right)$ such that $\left(p, e_{1}, \ldots, e_{m}\right) \in \mathcal{F}\left(M^{n}\right)$ and $\left(\varphi(p), e_{1}, \ldots, e_{m}\right) \in \mathcal{F}\left(\mathbb{E}^{m}\right)$. Then the natural projection $B(\varphi) \rightarrow M^{n}$ can be regarded as a principal bundle with fiber $O(n) \times S O(m-n)$, and $\tilde{\varphi}: B(\varphi) \rightarrow \mathcal{F}\left(\mathbb{E}^{m}\right)$ is naturally defined by $\tilde{\varphi}(b)=\left(\varphi(p), e_{1}, \ldots, e_{m}\right)$.

To avoid confusion, we shall use Einstein's convention on summation and also use the following ranges of indices:

$$
1 \leq i, j, k, \ldots \leq n ; \quad n+1 \leq r, s, t, \ldots \leq m ; \quad A, B, C \ldots, \ldots \leq m
$$

throughout this article, unless otherwise stated.

On $\mathcal{F}\left(\mathbb{E}^{m}\right)$, we introduce the 1 -forms $\theta^{A}, \theta_{A}^{B}$ defined by

$$
d p=\sum \theta^{A} e_{A}, \quad d e_{A}=\sum \theta_{A}^{B} e_{B}, \quad \theta_{A}^{B}=-\theta_{B}^{A} .
$$

Since $d^{2}=0$, it follows from (2.1) that

$$
d \theta^{A}=\sum \theta^{B} \wedge \theta_{B}^{A}, \quad d \theta_{B}^{A}=-\sum \theta_{C}^{A} \wedge \theta_{B}^{C},
$$

where $\wedge$ denotes the exterior product.

Let $\omega^{A}$ and $\omega_{B}^{A}$ denote the restrictions of the 1-forms $\theta^{A}$ and $\theta_{A}^{A}$ to $M^{n}$ via the immersion $\varphi$. Then we have $\omega^{r}=0$. Thus, we find from (2.1) and (2.2) that

$$
\begin{aligned}
& d \varphi=\sum \theta^{i} e_{i}, \quad d e_{m}=-\sum_{A=1}^{m-1} \omega_{A}^{m} e_{A}, \\
& d \omega^{i}=\sum \omega^{j} \wedge \omega_{j}^{i}, \quad d \omega_{j}^{i}=-\sum \omega_{k}^{i} \wedge \omega_{j}^{k}+\sum_{r} \omega_{i}^{r} \wedge \omega_{j}^{r},
\end{aligned}
$$

It was well-known that $\omega^{1}, \ldots, \omega^{n}$ are linearly independent 1 -forms on $M^{n}$ and the volume element of $M^{n}$ is given by $d v=\omega_{1} \wedge \ldots \wedge \omega_{n}$.

Since $0=d \omega^{r}=\sum \omega^{i} \wedge \omega_{i}^{s}$, Cartan's lemma implies that

$$
\omega_{i}^{r}=\sum h_{i j}^{r} \omega^{j}, \quad h_{i j}^{r}=h_{j i}^{r},
$$

where $h_{i j}^{r}$ are called the coefficients of the second fundamental form $h$. The eigenvalues $\kappa_{1}\left(p, e_{r}\right), \ldots, \kappa_{n}\left(p, e_{r}\right)$ of the symmetric matrix $\left(h_{i j}^{r}\right)$ via the second fundamental form are called the principal curvatures of $M^{n}$ at a point $\left(p, e_{r}\right) \in B_{1}(\varphi)$.

From (2.4) and (2.5) we obtain

$$
d \omega_{j}^{i}=\sum \omega_{i}^{k} \wedge \omega_{j}^{k}+\sum_{r, k, \ell}\left(h_{i k}^{r} h_{j \ell}^{r}-h_{i \ell}^{r} h_{j k}^{r}\right) \omega^{k} \wedge \omega^{\ell}
$$


The $\ell$-th mean curvature $K_{\ell}\left(p, e_{r}\right)$ at $\left(p, e_{r}\right)$ is defined by the elementary symmetric functions such that

$$
\left(\begin{array}{c}
n \\
\ell
\end{array}\right) K_{\ell}\left(p, e_{r}\right)=\sum \kappa_{1}\left(p, e_{r}\right) \cdots \kappa_{\ell}\left(p, e_{r}\right), \quad \ell=1, \ldots, n,
$$

where $\left(\begin{array}{c}n \\ \ell\end{array}\right)=n ! /(\ell !(n-\ell) !)$ denotes the binomial coefficients (cf. [1, 8, 9, 10, 14]). In particular, the $n$-th mean curvature $K_{n}(p, e)$ is well-known as the Lipschitz-Killing curvature at $(p, e)$.

Consider the principal bundle $B_{1}(\varphi) \rightarrow M^{n}$ with fiber $S_{p}^{m-n-1}$. Then

$$
d \sigma=\omega_{n+1}^{m} \wedge \cdots \wedge \omega_{m-1}^{m}
$$

is a differential form of degree $(m-n-1)$ on $B_{1}(\varphi)$ such that its restriction to the fiber $S_{p}^{m-n-1}$ of $B_{1}(\varphi)$ at a point $p \in M^{n}$ is the volume element of $S_{p}^{m-n-1}$. Therefore, $d v \wedge d \sigma$ is the volume element of the principal bundle $B_{1}(\varphi)$ (cf. $\left.[5,10]\right)$.

Also, it follows from (2.5) and (2.7) that we have

$$
\omega_{1}^{m} \wedge \cdots \wedge \omega_{n}^{m}=K_{n}\left(p, e_{m}\right) d v .
$$

Note that, for a hypersurface $M^{n}$ in $\mathbb{E}^{n+1}$, the fiber $S_{p}^{0}$ at a point $p \in M^{n}$ consists of two unit vectors at $p$; namely the unit outward normal vector and unit inner normal vector at $\varphi(p)$. Also, note that the $\ell$-th mean curvature satisfies

$$
K_{\ell}(p,-e)=(-1)^{\ell} K_{\ell}(p, e)
$$

for $\ell=0,1, \ldots, n$.

\section{G-total curvatures, g-Steiner, co-Steiner and normal points}

Now, we recall the notion of G-total curvatures from [4,7]. For a given immersion $\varphi: M^{n} \rightarrow \mathbb{E}^{m}$ of a bounded $n$-manifold $M^{n}$ into $\mathbb{E}^{m}$, let $\eta: B_{1}(\varphi) \rightarrow \mathbb{E}^{r}$ be a $\mathbb{E}^{r}$-valued function on the principal bundle $B_{1}(\varphi)$. In particular, if $r=1$, then $\eta$ is nothing but a real-value function on $B_{1}(\varphi)$.

For $\ell \in\{0,1,2, \ldots\}$ and $k \geq 0$, the integral

$$
G_{\ell}(\varphi, p, \eta, k)=\int_{e \in S_{p}^{m-n-1}} \eta(p, e)\left(K_{\ell}(p, e)\right)^{k} d \sigma,
$$

is called the $\ell$-th G-total curvature of rank $k$ at the point $p \in M^{n}$ with respect to the function $\eta$ if the right-hand-side of (3.1) exists (cf. [4, 7]).

An immersion $\varphi: M^{n} \rightarrow \mathbb{E}^{m}$ is called pseudo-flat if $G_{n}(\varphi, p, 1,1)=0$ for all $p \in M^{n}$ (cf. [4]). It follows from (2.9) that every immersion $\varphi: M^{n} \rightarrow \mathbb{E}^{m}$ of $M^{n}$ is always pseudo-flat whenever $n=\operatorname{dim} M^{n}$ is odd.

The integral

$$
T_{\ell}(\varphi, \eta, k)=\frac{1}{c_{m-1}} \int_{p \in M^{n}} G_{\ell}(\varphi, p, \eta, k) d v=\int_{(p, e) \in B(\varphi)} \eta(p, e)\left(K_{\ell}(p, e)\right)^{k} d v \wedge d \sigma
$$

is called the $\ell$-th G-total curvature with resect to $\eta$ if the right-hand-side of (3.2) exists (cf. [4, 7]).

For some special cases of G-total curvatures, we have the following.

- $T_{\ell}(\varphi, \eta, 0)$ is the ordinary $\mathbb{E}^{m}$-valued integral over $B_{1}(\varphi)$;

- $T_{\ell}(\varphi, 1,0)$ is the volume of $B_{1}(\varphi)$, where 1 denotes the constant function 1 on $B_{1}(\varphi)$;

- $T_{0}(\varphi, \varphi, 0)$ is the center of mass of $M^{n}$ in $\mathbb{E}^{m}$;

- If $M^{n}$ is a closed manifold, then $T_{n}(\varphi, 1,1)=\mathcal{X}\left(M^{n}\right)$, where $\mathcal{X}\left(M^{n}\right)$ denotes the Euler characteristic of $M^{n}$ (see [7, Proposition 6.4]);

- If $m=n+1$ and $n$ is even, then $\frac{1}{2} G_{n}(\varphi, p, 1,1)$ is the Gauss-Kronecker curvature of the hypersurface $M^{n}$ in $\mathbb{E}^{n+1}$ (cf. $\left.[2,6]\right)$.

For simplicity, we put

$$
T(\varphi)=T_{n}(\varphi, 1,1) \quad \text { and } \quad T(\varphi, \eta)=T_{n}(\varphi, \eta, 1) .
$$

For an immersion $\varphi: M^{n} \rightarrow \mathbb{E}^{m}$ and for $(p, e) \in B_{1}(\varphi)$, we define $g$-Steiner points, co-Steiner points and normal points of $\varphi$ via (3.2) and (3.3) as follows: 
- $g s(\varphi)=T(\varphi, \varphi)$ is called the $g$-Steiner point of $\varphi$;

- $\operatorname{cs}(\varphi)=T(\varphi,\langle\varphi, e\rangle e)$ is called the co-Steiner point of $\varphi$;

- $\mathbf{n}(\varphi)=T(\varphi, e)$ is called the normal point of $\varphi$,

where $\langle\varphi, e\rangle$ denotes the inner product for the $\mathbb{E}^{m}$-valued functions $\varphi$ and the unit normal vector $e$ on $M^{n}$.

Now, we prove the following two lemmas.

Lemma 3.1. If $\varphi: M^{n} \rightarrow \mathbb{E}^{m}$ is an immersion of a bounded manifold $M^{n}$ in $\mathbb{E}^{m}$, then the g-Steiner point $g s(\varphi)$ of $\varphi$ is given by

$$
g s(\varphi)=\frac{1}{c_{m-1}} \int_{\left(p, e_{m}\right) \in B_{1}(\varphi)} \varphi \omega_{1}^{m} \wedge \cdots \wedge \omega_{m-1}^{m} .
$$

Proof. Follows easily from the definition of g-Steiner point and Eqs. (2.8), (3.1) and (3.2).

Lemma 3.2. For an immersion $\varphi: M^{n} \rightarrow \mathbb{E}^{n+1}$ of an even-dimensional closed convex hypersurface $M^{n}$ in $\mathbb{E}^{n+1}$, the $g$-Steiner point $g s(\varphi)$ of $\varphi$ and the Steiner point $s\left(M^{n}\right)$ of $M^{n}$ defined by (1.1) are related by $g s(\varphi)=2 s\left(M^{n}\right)$.

Proof. Let $\varphi: M^{n} \rightarrow \mathbb{E}^{n+1}$ be the immersion of a closed convex hypersurface. Denote by $\mathrm{x}$ the position vector field of $M^{n}$ in $\mathbb{E}^{n+1}$. Then it follows from Eqs. (3.1), (3.2) and the definition of the g-Steiner point $g s(\varphi)$ of $\varphi$ that

$$
\begin{aligned}
g s(\varphi) & =\frac{1}{c_{n}} \int_{\left(p, e_{n+1}\right) \in B_{1}(\varphi)} \varphi \omega_{1}^{n+1} \wedge \cdots \wedge \omega_{n}^{n+1} \\
& =\frac{1}{c_{n}} \int_{p \in M^{n}} \varphi\left\{\int_{e \in S_{p}^{0}} K_{n}(p, e) d \sigma\right\} d v \\
& =\frac{2}{c_{n}} \int_{p \in M^{n}} \mathbf{x} K(p) d v
\end{aligned}
$$

where $K(p)$ is the Gauss-Kronecker curvature of $M^{n}$ at a point $p \in M^{n}, K_{n}(p, e)$ is the Lipschitz-Killing curvature at $(p, e) \in B_{1}(\varphi)$ and $\mathrm{x}$ is the position vector field of $\varphi: M^{n} \rightarrow \mathbb{E}^{n+1}$. Therefore, after comparing (3.5) with (1.1), we obtain $g s(\varphi)=2 s\left(M^{n}\right)$.

The next lemma is direct to verify.

Lemma 3.3. If $\varphi: M^{n} \rightarrow \mathbb{E}^{m}$ is an immersion of a bounded manifold $M^{n}$ in $\mathbb{E}^{m}$ and $\psi: \mathbb{E}^{m} \hookrightarrow \mathbb{E}^{\bar{m}}$ is an inclusion map, then we have $g s(\varphi)=g s(\bar{\varphi})$ with $\bar{\varphi}=\psi \circ \varphi$.

\section{Some properties of g-Steiner and co-Steiner points}

For g-Steiner points, we have the following.

Theorem 4.1. For a given immersion $\varphi: M^{n} \rightarrow \mathbb{E}^{m}$ of a bounded n-manifold $M^{n}$ into $\mathbb{E}^{m}$, the g-Steiner point gs $(\varphi)$ of $\varphi$ satisfies the following properties:

(a) If $a$ is a positive number and $\bar{\varphi}=a \varphi$ is the similarity transformation of $\varphi$ given by $(a \varphi)(p)=a(\varphi(p)), p \in M^{n}$, then we have $g s(a \varphi)=a(g s(\varphi))$;

(b) For a constant vector $c \in \mathbb{E}^{m}$, we have

$$
g s\left(\varphi_{c}\right)=g s(\varphi)+T(\varphi) c,
$$

where $\varphi_{c}=\varphi+c$ is the parallel translation of $\varphi$ given by $\varphi_{c}(p)=\varphi(p)+c$, and $T(\varphi)$ is the total Lipschitz-Killing curvature of $M^{n}$ defined by (3.3), i.e.,

$$
T(\varphi)=\frac{1}{c_{m-1}} \int_{\left(p, e_{m}\right) \in B_{1}(\varphi)} \omega_{1}^{m} \wedge \cdots \wedge \omega_{m-1}^{m} ;
$$

(c) If $\varphi: M^{n} \rightarrow \mathbb{E}^{m}$ is a pseudo-flat immersion, then $g s(\varphi)=0$. In particular, we have $g s(\varphi)=0$ whenever $M^{n}$ is of odd dimension; 
(d) If $\varphi: M^{n} \rightarrow \mathbb{E}^{m}$ and $\bar{\varphi}: \bar{M}^{\bar{n}} \rightarrow \mathbb{E}^{\bar{m}}$ are immersions of even-dimensional bounded manifolds $M^{n}$ and $\bar{M}^{\bar{n}}$ into $\mathbb{E}^{m}$ and $\mathbb{E}^{\bar{m}}$ respectively, then we have

$$
g s(\varphi \times \bar{\varphi})=(T(\bar{\varphi}) g s(\varphi), T(\varphi) g s(\bar{\varphi})),
$$

where $\varphi \times \bar{\varphi}$ is the product immersion of $\varphi$ and $\bar{\varphi}$ given by $M^{n} \times \bar{M}^{\bar{n}} \ni(p, \bar{p}) \mapsto(\varphi(p), \bar{\varphi}(\bar{p})) \in \mathbb{E}^{m} \times \mathbb{E}^{\bar{m}}$.

Proof. (a) Let $a \varphi$ be a similarity transformation of $\varphi$. Then, the definition of $B_{1}(\varphi)$ yields $B_{1}(a \varphi)=B_{1}(\varphi)$. For a given point $\left(p, e_{1}, \ldots, e_{m}\right) \in B(a \varphi)$, we put

$$
d(a \varphi)=\sum \bar{\omega}^{i} e_{i}, \quad \bar{\omega}_{A}^{m}=\left\langle d e_{m}, e_{A}\right\rangle
$$

and let $\omega^{i}, \omega_{A}^{m}$ denote the corresponding forms on $B(\varphi)$. Then we obtain

$$
\bar{\omega}_{1}^{m} \wedge \cdots \wedge \bar{\omega}_{m-1}^{m}=\omega_{1}^{m} \wedge \cdots \wedge \omega_{m-1}^{m} .
$$

Consequently, we obtain from the definition of g-Steiner points and Lemma 3.1 that

$$
\begin{aligned}
g s(a \varphi) & =\frac{1}{c_{m-1}} \int_{\left(p, e_{m}\right) \in B_{1}(a \varphi)}(a \varphi) \bar{\omega}_{1}^{m} \wedge \cdots \wedge \bar{\omega}_{m-1}^{m} \\
& =\frac{a}{c_{m-1}} \int_{\left(p, e_{m}\right) \in B_{1}(\varphi)} \varphi \omega_{1}^{m} \wedge \cdots \wedge \omega_{m-1}^{m} \\
& =a(g s(\varphi)) .
\end{aligned}
$$

(b) For a given vector $c \in \mathbb{E}^{m}$, we have $B_{1}(\varphi)=B_{1}\left(\varphi_{c}\right)$. Thus, if we denote by $\bar{\omega}$ the form for $\varphi_{c}$ corresponding to a form $\omega$ for $\varphi$, then we obtain (4.3) as well. Therefore

$$
\begin{aligned}
g s\left(\varphi_{c}\right) & =\frac{1}{c_{m-1}} \int_{\left(p, e_{m}\right) \in B_{1}(\varphi+c)}(\varphi+c) \bar{\omega}_{1}^{m} \wedge \cdots \wedge \bar{\omega}_{m-1}^{m} \\
& =\frac{1}{c_{m-1}} \int_{\left(p, e_{m}\right) \in B_{1}(\varphi)} \varphi \omega_{1}^{m} \wedge \cdots \wedge \omega_{m-1}^{m}+\frac{c}{c_{m-1}} \int_{\left(p, e_{m}\right) \in B_{1}(\varphi)} \omega_{1}^{m} \wedge \cdots \wedge \omega_{m-1}^{m} \\
& =g s(\varphi)+\frac{c}{c_{m-1}} \int_{\left(p, e_{m}\right) \in B_{1}(\varphi)} \omega_{1}^{m} \wedge \cdots \wedge \omega_{m-1}^{m} \\
& =g s(\varphi)+T(\varphi) c
\end{aligned}
$$

where $T(\varphi)$ is defined by $(4.1)$.

(c) The first part of statement (c) follows from Eq. (2.8) and Lemma 3.2. And the second part of statement (c) follows from (2.9).

(d) Let $\varphi: M^{n} \rightarrow \mathbb{E}^{m}$ and $\varphi^{\prime}: M^{n^{\prime}} \rightarrow \mathbb{E}^{m^{\prime}}$ be immersions of bounded manifolds $M^{n}$ and $M^{n^{\prime}}$ into $\mathbb{E}^{m}$ and $\mathbb{E}^{m^{\prime}}$ respectively. According to Lemma 3.3, to prove statement (d), without loss of generality, we may assume $m$ and $\bar{m}$ are both odd.

Let $B(\varphi \times \bar{\varphi})$ be the bundle space over $M^{n} \times \bar{M}^{\bar{n}}$ consisting of

$$
\left((p, \bar{p}), e_{1}, \ldots, e_{n}, \bar{e}_{1}, \ldots, \bar{e}_{\bar{n}}, e_{n+1}, \ldots, e_{m}, \bar{e}_{\bar{n}+1}, \ldots, \bar{e}_{\bar{m}}\right)
$$

such that $\left(p, e_{1}, \ldots, e_{m}\right) \in B(\varphi)$ and $\left(\bar{p}, \bar{e}_{1}, \ldots, \bar{e}_{\bar{m}}\right) \in B(\bar{\varphi})$. Let us consider the following two unit normal vector fields of $M^{n} \times \bar{M}^{\bar{n}}$ in $\mathbb{E}^{m} \times \mathbb{E}^{\bar{m}}$ given by

$$
\tilde{e}_{m+\bar{m}-1}=-\sin \theta e_{m}+\cos \theta \bar{e}_{\bar{m}}, \quad \tilde{e}_{m+\bar{m}}=\cos \theta e_{m}+\sin \theta \bar{e}_{\bar{m}}
$$

over $M^{n} \times \bar{M}^{\bar{n}}$. Then we have

$$
d \tilde{e}_{m+\bar{m}}=\cos \theta d e_{m}+\sin \theta d \bar{e}_{\bar{m}}+\tilde{e}_{m+\bar{m}-1} d \theta .
$$

If $\tilde{\omega}_{\alpha}^{\beta}(\alpha, \beta=1, \ldots, m+\bar{m})$ denote the connection forms associated with the frame

$$
\left((p, \bar{p}), e_{1}, \ldots, e_{n}, \bar{e}_{1}, \ldots, \bar{e}_{\bar{n}}, e_{n+1}, \ldots, e_{m-1}, e_{m+\bar{m}-1}, \bar{e}_{\bar{n}+1}, \ldots, \bar{e}_{\bar{m}-1}, \bar{e}_{m+\bar{m}}\right)
$$


then we find

$$
\begin{aligned}
& \tilde{\omega}_{i}^{m+\bar{m}}=\cos \theta \omega_{i}^{m}, \quad \tilde{\omega}_{\bar{i}}^{m+\bar{m}}=\sin \theta \omega_{\bar{i}}^{\bar{m}}, \quad i=1, \ldots, n ; \quad \bar{i}=1, \ldots, \bar{n} \\
& \tilde{\omega}_{r}^{m+\bar{m}}=\cos \theta \omega_{r}^{m}, \quad \tilde{\omega}_{\bar{r}}^{m+\bar{m}}=\cos \theta \omega_{\bar{r}}^{\bar{m}}, \quad r=n+1, \ldots, m-1 ; \quad \bar{r}=\bar{n}+1, \ldots, \bar{m}-1 ; \\
& \tilde{\omega}_{m+\bar{m}-1}^{m+\bar{m}}=d \theta .
\end{aligned}
$$

Now, it follows from (4.6), (4.7) and (4.8) that

$$
\begin{aligned}
d \tilde{v} \wedge d \tilde{\sigma}= & \tilde{\omega}_{1}^{m+\bar{m}} \wedge \cdots \wedge \tilde{\omega}_{m+\bar{m}-1}^{m+\bar{m}} \\
=(\cos \theta)^{m-1}(\sin \theta)^{\bar{m}-1} K_{n}\left(p, e_{m}\right) \bar{K}_{\bar{n}}\left(\bar{p}, \bar{e}_{\bar{m}}\right) & \\
& \quad \times d v \wedge d \bar{v} \wedge \omega_{n+1}^{m} \wedge \cdots \wedge \omega_{m-1}^{m} \wedge \omega_{\bar{n}+1}^{m} \wedge \cdots \wedge \omega_{\bar{m}-1}^{\bar{m}} \wedge d \theta .
\end{aligned}
$$

Consequently,

$$
\begin{aligned}
g s(\varphi \times \bar{\varphi}) & =\frac{1}{c_{m+\bar{m}-1}} \int_{e_{m+\bar{m}} \in B_{1}(\varphi \times \bar{\varphi})}(\varphi \times \bar{\varphi}) \tilde{\omega}_{1}^{m+\bar{m}} \wedge \cdots \wedge \tilde{\omega}_{m+\bar{m}-1}^{m+\bar{m}} \\
= & \frac{1}{c_{m+\bar{m}-1}} \int_{e_{m+\bar{m}} \in B_{1}(\varphi \times \bar{\varphi})}(\varphi \times \bar{\varphi})\left(\cos ^{m-1} \theta\right)\left(\sin ^{\bar{m}-1} \theta\right) \\
= & (T(\bar{\varphi}) g s(\varphi), T(\varphi) g s(\bar{\varphi})),
\end{aligned}
$$

where we have used (cf. Formula (4.6) of [9, page 135])

$$
\int_{S^{1}}\left(\cos ^{p} \theta \sin ^{q} \theta\right) d \theta=\frac{2 \Gamma((1+p) / 2) \Gamma((1+q) / 2)}{\Gamma((2+p+q) / 2))}
$$

for even integers $p, q \geq 0$ and

$$
c_{n}=\frac{(n+1) \pi^{(n+1) / 2}}{\Gamma((n+3) / 2)} .
$$

The next three corollaries follow from Theorem 4.1 and the fact that the total Gauss-Kronecker curvature of $\varphi$ satisfies $T(\varphi)=c_{m-1} \mathcal{X}\left(M^{n}\right)$ (cf. [7, Proposition 6.4]).

Corollary 4.1. Let $\varphi: M^{n} \rightarrow \mathbb{E}^{m}$ be an immersion of an even-dimensional closed oriented manifold $M^{n}$ into $\mathbb{E}^{m}$. Then the Euler characteristic of $M^{n}$ satisfies $\mathcal{X}\left(M^{n}\right)=0$ if and only if the g-Steiner point of $\varphi$ is invariant under translations.

Corollary 4.2. Let $\varphi: M^{n} \rightarrow \mathbb{E}^{m}$ and $\bar{\varphi}: \bar{M}^{\bar{n}} \rightarrow \mathbb{E}^{\bar{m}}$ be immersions of even-dimensional closed oriented manifolds $M^{n}$ and $\bar{M}^{\bar{n}}$ into $\mathbb{E}^{m}$ and $\mathbb{E}^{\bar{m}}$ respectively. If $\mathcal{X}\left(M^{n}\right)=\mathcal{X}\left(\bar{M}^{\bar{n}}\right)=0$, then $g s(\varphi \times \bar{\varphi})=0$.

Corollary 4.3. Let $M^{n}$ be an oriented closed manifold with $\mathcal{X}\left(M^{n}\right) \neq 0$. If $M^{n}$ is immersible into $\mathbb{E}^{m}$, then for any $c \in \mathbb{E}^{m}$, there exists an immersion of $\varphi: M^{n} \rightarrow \mathbb{E}^{m}$ such that $g s(\varphi)=c$.

We have the following properties for co-Steiner points.

Theorem 4.2. For a given immersion $\varphi: M^{n} \rightarrow \mathbb{E}^{m}$ of a bounded n-manifold $M^{n}$ into $\mathbb{E}^{m}$, the co-Steiner point $\operatorname{cs}(\varphi)$ of $\varphi$ satisfies the following properties:

(a) If a is a positive number, then we have $\operatorname{cs}(a \varphi)=a(\operatorname{cs}(\varphi))$;

(b) For a constant vector $c \in \mathbb{E}^{m}$, the translation $\varphi_{c}$ of $\varphi$ satisfies

$$
\operatorname{cs}\left(\varphi_{c}\right)=c s(\varphi)+T(\varphi,\langle c, e\rangle e),
$$

where $T(\varphi, c)$ is the $n$-th G-total curvature of $M^{n}$ defined by

$$
T(\varphi,\langle c, e\rangle e)=\frac{1}{c_{m-1}} \int_{(p, e) \in B_{1}(\varphi)}\langle c, e\rangle e \omega_{1}^{m} \wedge \cdots \wedge \omega_{m-1}^{m}, \quad e=e_{m} ;
$$

(c) If $\operatorname{dim} M^{n}$ is odd, then $\operatorname{cs}(\varphi)=0$.

Since this theorem can be proved in similar way as Theorem 4.1, so we omit its proof. 


\section{Some properties of normal points}

Let $v_{1}, \ldots, v_{m-1}, v$ be $m$ vector in $\mathbb{E}^{m}$ and let $v_{1} \times \cdots \times v_{m-1}$ denote the vector product of $v_{1}, \ldots, v_{m-1}$. Then we get

$$
v \cdot\left(v_{1} \times \cdots \times v_{m-1}\right)=(-1)^{m-1}\left|v, v_{1}, \cdots, v_{m-1}\right|,
$$

where $\left|v, v_{1}, \cdots, v_{m-1}\right|$ denotes the determinant of $v, v_{1}, \ldots, v_{m-1}$. From (5.1) we find

$$
e_{1} \times \cdots \times \widehat{e}_{\alpha} \times \cdots \times e_{m}=(-1)^{m+\alpha} e_{\alpha}
$$

where the roof $\hat{\cdot}$ means the omitted term.

Let $\varphi: M^{n} \rightarrow \mathbb{E}^{m}$ be an immersion of a bounded $n$-manifold $M^{n}$ into $\mathbb{E}^{m}$. As before, we denote by $B(\varphi)$ the bundle space consists of all $\left(p, e_{1}, \ldots, e_{m}\right)$ over $M^{n}$ defined as in Section 2. Now, we define a $\mathbb{E}^{m}$-valued $(m-1)$-form $\Omega$ given by

$$
\Omega=\frac{1}{(m-1) c_{m-1}} \sum_{\alpha=1}^{m-1}(-1)^{\alpha}\left(\omega_{1}^{m} \wedge \cdots \wedge \widehat{\omega}_{\alpha}^{m} \wedge \cdots \wedge \omega_{m-1}^{m}\right) e_{\alpha} .
$$

Proposition 5.1. For a given immersion $\varphi: M^{n} \rightarrow \mathbb{E}^{m}$ of a bounded n-manifold $M^{n}$ into $\mathbb{E}^{m}$, the normal point $\mathbf{n}(\varphi)$ of $\varphi$ satisfies

$$
\mathbf{n}(\varphi)=\int_{\partial B_{1}(\varphi)} \Omega
$$

where $\partial B_{1}(\varphi)$ denotes the boundary of $B_{1}(\varphi)$.

Proof. For a given immersion $\varphi: M^{n} \rightarrow \mathbb{E}^{m}$ of a bounded $n$-manifold $M^{n}$ into $\mathbb{E}^{m}$, let $[\cdot, \ldots, \cdot]$ denote the combining operation of the vector product of $\mathbb{E}^{m}$ with the exterior product.

If we denote $e_{m}$ by $e$, then we have

$$
\begin{aligned}
\overbrace{d e, \ldots, d e, e]}^{m-2 \text { times }} & =(-1)^{m}\left[\sum \omega_{\alpha_{1}}^{m} e_{\alpha_{1}}, \ldots, \sum \omega_{\alpha_{m-2}}^{m} e_{\alpha_{m-2}}, e\right] \\
& =(-1)^{m} \sum \omega_{\alpha_{1}}^{m} \wedge \cdots \wedge \omega_{\alpha_{m-2}}^{m}\left[e_{\alpha_{1}}, \ldots, e_{\alpha_{m-2}}, e\right] \\
& =(-1)^{m}(m-2) ! \sum_{\alpha=1}^{m-1} \omega_{1}^{m} \wedge \cdots \wedge \widehat{\omega}_{\alpha}^{m} \wedge \ldots \wedge \omega_{m-1}^{m}\left[e_{1}, \ldots, \widehat{e}_{\alpha}, \ldots, e_{m-1}, e\right] \\
& =(m-2) ! \sum_{\alpha=1}^{m-1}(-1)^{\alpha}\left(\omega_{1}^{m} \wedge \cdots \wedge \widehat{\omega}_{\alpha}^{m} \wedge \cdots \wedge \omega_{m-1}^{m}\right) e_{\alpha},
\end{aligned}
$$

Combining this with (5.3) gives

$$
\Omega=\frac{1}{(m-1) ! c_{m-1}}[\overbrace{d e, \ldots, d e}^{m-2 \text { times }}, e]
$$

Since

$$
d(\overbrace{d e, \ldots, d e}^{m-2 \text { times }}, e])=(-1)^{m}[\overbrace{d e, \ldots, d e}^{m-1 \text { times }}]
$$

it follows from (5.5) that

$$
\begin{aligned}
d \Omega & =\frac{(-1)^{m}}{(m-1) ! c_{m-1}}[\overbrace{d e, \ldots, d e}^{m-1 \text { times }}] \\
& =-\frac{1}{(m-1) ! c_{m-1}}\left[\sum \omega_{\alpha_{1}}^{m} e_{\alpha_{1}}, \ldots, \sum \omega_{\alpha_{m-1}}^{m} e_{\alpha_{m-1}}\right] \\
& =-\frac{1}{(m-1) ! c_{m-1}} \sum \omega_{\alpha_{1}}^{m} \wedge \cdots \wedge \omega_{\alpha_{m-1}}^{m}\left[e_{\alpha_{1}}, \ldots, e_{\alpha_{m-1}}\right] \\
& =\frac{1}{c_{m-1}}\left(e \omega_{1}^{m} \wedge \cdots \wedge \omega_{m-1}^{m}\right) .
\end{aligned}
$$


Consequently, after applying Stokes' theorem we obtain

$$
\begin{aligned}
\mathbf{n}(\varphi) & =\frac{1}{c_{m-1}} T(\varphi, e) \\
& =\frac{1}{c_{m-1}} \int_{(p, e) \in B_{1}(\varphi)} e K_{n}(p, e) d v \wedge d \sigma \\
& =\frac{1}{c_{m-1}} \int_{(p, e) \in B_{1}(\varphi)} e \omega_{1}^{m} \wedge \cdots \wedge \omega_{m-1}^{m} \\
& =\int_{B_{1}(\varphi)} d \Omega=\int_{\partial B_{1}(\varphi)} \Omega,
\end{aligned}
$$

which proves the Proposition.

For the normal point $\mathbf{n}(\varphi)$ of $\varphi$, we have the following.

Theorem 5.1. For a given immersion $\varphi: M^{n} \rightarrow \mathbb{E}^{m}$ of a bounded n-manifold $M^{n}$ into $\mathbb{E}^{m}$, the normal point of $\varphi$ satisfies the following three properties:

(a) The normal point is invariant under similarity transformations;

(b) The normal point is invariant under translations;

(c) If $M^{n}$ is of even dimension or $M^{n}$ is a closed manifold, then we have $\mathbf{n}(\varphi)=0$.

Proof. Since statements (a) and (b) can be proved in similar ways as the proofs of statements (a) and (b) of Theorem 4.1, so we omit them.

(c) First, if $\operatorname{dim} M^{n}$ is even, then the Lipschitz-Killing curvature satisfies $K(p, e)=K(p,-e)$, Thus, by the definition of the normal point $\mathbf{n}(\varphi)$ and the symmetry of the fiber $S_{p}^{m-1}$ over $p$, we easily see that the G-total curvature $G_{n}(\varphi, p, e, 1)=0$ for each $p \in M^{n}$. Hence we get $\mathbf{n}(\varphi)=0$.

Second, if $M^{n}$ is a closed manifold, then statement (c) follows from Proposition 5.1.

\section{A link between g-Steiner and co-Steiner points}

The next theorem provides a link between g-Steiner and co-Steiner points.

Theorem 6.1. Let $\varphi: M^{n} \rightarrow \mathbb{E}^{m}$ be an immersion of a closed manifold $M^{n}$ into $\mathbb{E}^{m}$. Then the g-Steiner and co-Steiner points of $\varphi$ are related by $g s(\varphi)=m(\operatorname{cs}(\varphi))$.

Proof. For an immersion $\varphi: M^{n} \rightarrow \mathbb{E}^{m}$ of a closed manifold $M^{n}$ into $\mathbb{E}^{m}$, let $\Omega$ be defined as (5.3). Then it follows from (2.3) and (5.5) that

$$
\begin{aligned}
d\left(\left\langle\varphi, e_{m}\right\rangle \Omega\right) & =\left(d\left\langle\varphi, e_{m}\right\rangle\right) \wedge \Omega+\left\langle\varphi, e_{m}\right\rangle d \Omega \\
& =\left(\left\langle\varphi, d e_{m}\right\rangle\right) \wedge \Omega+\left\langle\varphi, e_{m}\right\rangle d \Omega \\
& =-\frac{1}{(m-1) c_{m-1}} \sum_{\alpha}^{m-1}\left\langle\varphi, e_{\alpha}\right\rangle \omega_{\alpha}^{m} \wedge \sum_{\beta=1}^{m-1}(-1)^{\beta}\left(\omega_{1}^{m} \wedge \cdots \wedge \widehat{\omega}_{\beta}^{m} \wedge \cdots \wedge \omega_{m-1}^{m}\right) e_{\beta} \\
& \quad+\left\langle\varphi, e_{m}\right\rangle d \Omega \\
= & -\frac{1}{(m-1) c_{m-1}} \sum_{\alpha}^{m-1}\left\langle\varphi, e_{\alpha}\right\rangle e_{\alpha}\left(\omega_{1}^{m} \wedge \cdots \wedge \omega_{m-1}^{m}\right)+\left\langle\varphi, e_{m}\right\rangle d \Omega \\
= & -\frac{1}{(m-1) c_{m-1}}\left(\varphi-\left\langle\varphi, e_{m}\right\rangle e_{m}\right)\left(\omega_{1}^{m} \wedge \cdots \wedge \omega_{m-1}^{m}\right)+\left\langle\varphi, e_{m}\right\rangle d \Omega .
\end{aligned}
$$

After combining (6.1) with (5.6) we find

$$
\begin{aligned}
d(\langle\varphi, e\rangle \Omega) & =-\frac{1}{(m-1) c_{m-1}}(\varphi-\langle\varphi, e\rangle e)\left(\omega_{1}^{m} \wedge \cdots \wedge \omega_{m-1}^{m}\right)+\frac{1}{c_{m-1}}(\langle\varphi, e\rangle e) \omega_{1}^{m} \wedge \cdots \wedge \omega_{m-1}^{m} \\
& =\frac{1}{(m-1) c_{m-1}}\left\{m(\langle\varphi, e\rangle e) \omega_{1}^{m} \wedge \cdots \wedge \omega_{m-1}^{m}-\varphi\left(\omega_{1}^{m} \wedge \cdots \wedge \omega_{m-1}^{m}\right)\right\}
\end{aligned}
$$

with $e=e_{m}$. Consequently, after applying Stokes' theorem we obtain $\operatorname{sg}(\varphi)=m(\operatorname{cs}(\varphi))$ since $M$ is a closed manifold. 
Corollary 6.1. Let $\varphi: M^{n} \rightarrow \mathbb{E}^{m}$ and $\bar{\varphi}: \bar{M}^{\bar{n}} \rightarrow \mathbb{E}^{\bar{m}}$ be two immersions of two even-dimensional closed manifolds $M^{n}$ and $\bar{M}^{\bar{n}}$ into $\mathbb{E}^{m}$ and $\mathbb{E}^{\bar{m}}$, respectively. Then we have

$$
c s(\varphi \times \bar{\varphi})=\frac{1}{m+\bar{m}}(T(\bar{\varphi}) g s(\varphi), T(\varphi) g s(\bar{\varphi})) .
$$

where $\varphi \times \bar{\varphi}$ is the product immersion of $\varphi$ and $\bar{\varphi}$

Proof. Follows from Theorem 6.1 and statement (d) of Theorem 4.1.

\section{7. g-Steiner and co-Steiner points of higher order}

Let $\varphi: M^{n} \rightarrow \mathbb{E}^{m}$ be an immersion of a bounded $n$-manifold $M^{n}$ into $\mathbb{E}^{m}$. For $k=1,2, \ldots, n$, let us introduce the following notion of Steiner point of order $k$ of $\varphi$.

Definition 7.1. The point $g s^{k}(\varphi)=T\left(\varphi,\langle\varphi, e\rangle^{k-1} \varphi\right)$ is called the $g$-Steiner point of order $k$.

Similarly, we make the following.

Definition 7.2. The point $c s^{k}(\varphi)=T\left(\varphi,\langle\varphi, e\rangle^{k-1} e\right)$ is called the co-Steiner point of order $k$.

Clearly, the g-Steiner (respectively, co-Steiner) point of order 1 is nothing but the g-Steiner (respectively, co-Steiner) point defined in Section 3.

The g-Steiner and co-Steiner points of higher order are related by the following.

Theorem 7.1. Let $\varphi: M^{n} \rightarrow \mathbb{E}^{m}$ be an immersion of a bounded $n$-manifold $M^{n}$ into $\mathbb{E}^{m}$. Then we have:

$$
k\left(g s^{k}(\varphi)\right)=(m+k-1) c s^{k}(\varphi)+\frac{1}{c_{m-1}} \int_{\partial B(\varphi)}\langle\varphi, e\rangle^{k} \Omega .
$$

Proof. For an immersion $\varphi: M^{n} \rightarrow \mathbb{E}^{m}$ of a bounded manifold $M^{n}$ into $\mathbb{E}^{m}$, let $\Omega$ be defined as in (5.3). Then it follows from (2.3) and (5.5) that

$$
\begin{aligned}
d\left(\left\langle\varphi, e_{m}\right\rangle^{k} \Omega\right)= & \left(d\left\langle\varphi, e_{m}\right\rangle^{k}\right) \wedge \Omega+\left\langle\varphi, e_{m}\right\rangle^{k} d \Omega \\
= & k\left(\left\langle\varphi, d e_{m}\right\rangle^{k-1}\right) \wedge \Omega+\left\langle\varphi, e_{m}\right\rangle^{k} d \Omega \\
= & -\frac{k\left\langle\varphi, e_{m}\right\rangle^{k-1}}{(m-1) c_{m-1}} \sum_{\alpha}^{m-1}\left\langle\varphi, e_{\alpha}\right\rangle \omega_{\alpha}^{m} \wedge \sum_{\beta=1}^{m-1}(-1)^{\beta}\left(\omega_{1}^{m} \wedge \cdots \wedge \widehat{\omega}_{\beta}^{m} \wedge \cdots \wedge \omega_{m-1}^{m}\right) e_{\beta} \\
& \quad+\left\langle\varphi, e_{m}\right\rangle^{k} d \Omega \\
& =-\frac{k\left\langle\varphi, e_{m}\right\rangle^{k-1}}{(m-1) c_{m-1}} \sum_{\alpha}^{m-1}\left\langle\varphi, e_{\alpha}\right\rangle e_{\alpha}\left(\omega_{1}^{m} \wedge \cdots \wedge \omega_{m-1}^{m}\right)+\left\langle\varphi, e_{m}\right\rangle^{k} d \Omega \\
& =-\frac{k\left\langle\varphi, e_{m}\right\rangle^{k-1}}{(m-1) c_{m-1}}\left(\varphi-\left\langle\varphi, e_{m}\right\rangle e_{m}\right)\left(\omega_{1}^{m} \wedge \cdots \wedge \omega_{m-1}^{m}\right)+\left\langle\varphi, e_{m}\right\rangle^{k} d \Omega .
\end{aligned}
$$

After combining (7.2) with (5.6) we obtain

$$
\begin{aligned}
d\left(\langle\varphi, e\rangle^{k} \Omega\right) & =-\frac{k\left\langle\varphi, e_{m}\right\rangle^{k-1}}{(m-1) c_{m-1}}(\varphi-\langle\varphi, e\rangle e)\left(\omega_{1}^{m} \wedge \cdots \wedge \omega_{m-1}^{m}\right)+\langle\varphi, e\rangle^{k} d \Omega . \\
& =-\frac{k\left\langle\varphi, e_{m}\right\rangle^{k-1}}{(m-1) c_{m-1}}(\varphi-\langle\varphi, e\rangle e)\left(\omega_{1}^{m} \wedge \cdots \wedge \omega_{m-1}^{m}\right)+\frac{1}{c_{m-1}}\left(\langle\varphi, e\rangle^{k} e\right) \omega_{1}^{m} \wedge \cdots \wedge \omega_{m-1}^{m} \\
& =\frac{\left\langle\varphi, e_{m}\right\rangle^{k-1}}{(m-1) c_{m-1}}\left\{(m+k-1)(\langle\varphi, e\rangle e) \omega_{1}^{m} \wedge \cdots \wedge \omega_{m-1}^{m}-k \varphi\left(\omega_{1}^{m} \wedge \cdots \wedge \omega_{m-1}^{m}\right)\right\},
\end{aligned}
$$

with $e=e_{m}$. Consequently, after applying Stokes' theorem to (7.3) we obtain (7.1). 
The next corollary is an immediate consequence of Theorem 7.1.

Corollary 7.1. Let $\varphi: M^{n} \rightarrow \mathbb{E}^{m}$ be an immersion of a closed manifold $M^{n}$ into $\mathbb{E}^{m}$. Then the g-Steiner and co-Steiner points of order $k$ are related by

$$
g s^{k}(\varphi)=\frac{m+k-1}{k} c s^{k}(\varphi)
$$

for $k=2, \ldots, n$.

\section{References}

[1] Chen, B.-Y.: On the total absolute curvature of manifolds immersed in Riemannian manifold. Kodai Math. Sem. Rep. 19, 299-311 (1967)

[2] Chen, B.-Y.: Some integral formulas of the Gauss-Kronecker curvature. Kodai Math. Sem. Rep. 20, 410-413 (1968).

[3] Chen, B.-Y.: Notes on the G-Gauss-Kronecker curvature. Nanta Math. 2, 47-53 (1968).

[4] Chen, B.-Y.: On the G-total curvature and topology of immersed manifolds. Thesis (Ph.D.)-University of Notre Dame (1970).

[5] Chen, B.-Y.: On the total curvature of immersed manifolds. I. An inequality of Fenchel-Borsuk-Willmore. Amer. J. Math. 93, 148-162 (1971).

[6] Chen, B.-Y.: On an integral formula of Gauss-Bonnet-Grotemeyer. Proc. Amer. Math. Soc. 28, 208-212 (1971).

[7] Chen, B.-Y.: G-total curvature of immersed manifolds. J. Differential Geometry 7, 371-391 (1972).

[8] Chen, B.-Y.: Pseudo-Riemannian manifolds, $\delta$-invariants and applications. World Scientific Publishing, Hackensack, NJ (2011).

[9] Chen, B.-Y.: Geometry of Submanifolds. 2nd Edition, Dover Publications, Mineola, NY, (2019).

[10] Chern, S.-S., Lashof, R. K.: On the total curvature of immersed manifolds. Amer. J. Math. 79, 306-318 (1957).

[11] Flanders, H.: The Steiner point of a closed hypersurface. Mathematika 13, 181-188(1966).

[12] Kamma, L., Nutov, Z.: Approximating survivable networks with minimum number of Steiner points. Networks 60(4), 245-252 (2012).

[13] Meyer, W. J.: Characterization of the Steiner point. Pacific J. Math. 35, 717-725 (1970).

[14] Otsuki T.: On the total curvature of surfaces in Euclidean spaces. Japanese J. Math. 35, 61-71 (1966).

[15] Pechersky, S.: The Steiner point of a convex set and the cooperative games solutions. ICM2002GTA (Qingdao), 637-641, Qingdao Publ. House, Qingdao (2002).

[16] Schneider, R.: On Steiner points of convex bodies. Israel J. Math. 9, 241-249 (1971).

[17] Shephard, G. C.: The Steiner point of a convex polytope. Canadian J. Math. 18, 1294-1300 (1966).

[18] Shephard, G. C.: A uniqueness theorem for the Steiner point of a convex region. J. London Math. Soc. 43, $439-444$ (1968).

[19] Su, B.: On Steiner's curvature-centroid. Japanese J. Math. 4, 195-201 (1927).

[20] Steiner, J.: Von dem Krummungsschwerpunkt ebener Kurven. J. Reine Angew. Math. 21, 101-133 (1838).

\section{Affiliations}

\section{B.-Y. CHEN}

AdDREss: Department of Mathematics, Michigan State University, 619 Red Cedar Road, East Lansing, Michigan 48824-1027, U.S.A.

E-MAIL: chenb@msu.edu

ORCID ID: orcid.org/0000-0002-1270-094X 